to un plasmide IncN che conferisce la resistenza a tetraciclina e streptomicina ampiamente diffuso in ceppi di $S$. Blockley di origine aviaria. Un elemento trasponibile ritrovato prevalantemente nel sierotipo Hadar era stato precedente descritto solo in batteri patogeni delle piante.

I risultati di questo studio dimostrano l'importanza del trasferimento orizzontale tra batteri di origine diversa nell'acquisizione della resistenza agli antibiotici.

\section{M041 \\ DUE ANNI DI OSSERVAZIONE DELLE BATTERIEMIE: EPIDEMIOLOGIA ED EZIOLOGIA DEGLI ISOLATI IN RELAZIONE A DIVERSE AREE DI DEGENZA.}

\section{Usiglio D., Casini Lemmi M., Chisci R., Stepinska D., Lanata M., Mori M. \\ Struttura Complessa di Analisi Chimico-Cliniche e Microbiologiche "E. O. Ospedali Galliera", Genova.}

Obiettivi: Dato l'elevato valore predittivo positivo e l'importanza, sia come strumento di sorveglianza delle infezioni ospedaliere che come indice di qualità dell'operatività interna, è stata svolta un'analisi retrospettiva dei dati riguardanti l'emocolture eseguite nell'ultimo biennio (2001-2002). A questo scopo sono stati utilizzati indici consolidati e confrontabili nel monitoraggio epidemiologico ed eziologico delle batteriemie, evidenziando soprattutto le differenze tra diverse aree di degenza.

Metodi: Sono state individuate tre aree di degenza: medicina (A.med), chirurgia (A.chir) e UTI. Le Batteriemie considerate significative erano quelle relative all'isolamento da emocoltura di un reale patogeno in relazione al numero dei prelievi eseguiti e alle evidenze cliniche. Sono state definite infezioni ospedaliere le batteriemie significative insorte almeno 72 ore dopo il ricovero ospedaliero, non manifeste clinicamente ne in incubazione al momento dell'ammissione. Per l'incubazione dei flaconi da emocoltura è stato utilizzato il sistema BacT-Alert (Biomerieux).

Risultati: Sono stati studiati complessivamente 2735 episodi infettivi pari a 50,4 episodi/1000 ricoveri. Sono state diagnosticate 335 batteriemie significative pari al 12,2\% degli episodi studiati. Le batteriemie polimicrobiche rappresentano il $2,7 \%$. Le pseudobatteriemie sono risultate pari al $10,3 \%$. Il tasso medio di batteriemie significative è stato di $6,6 / 1000$ ricoveri, di cui 2,8 ospedaliere e 3,8 comunitarie (con significative differenze tra le diverse aree di degenza). Complessivamente si è osservata una prevalenza delle batteriemie comunitarie pari al 57,3\%, le batteriemie ospedaliere $(42.7 \%)$ sono prevalenti nell'A.chir e UTI. Sono stati individuati 344 microrganismi responsabili di batteriemie, nelle batteriemie comunitarie prevalgono i gram negativi (54.5\%), nelle batteriemie ospedaliere prevalgono i gram positivi (54\%) e i lieviti (11.5\%) soprattutto nell'A.chir e UTI.

Conclusioni: I rilievi epidemiologici, in termini di numero di episodi infettivi e batteriemie significative in relazione al numero dei ricoveri, sono confrontabili a quelli osservati in studi analoghi nazionali e internazionali. Il preoccupante dato delle pseudobatteriemie ha sollecitato interventi mirati del CIO. I dati eziologici hanno messo in risalto le differenze tra diverse aree di degenza e il ruolo emergente degli stafilococchi coagulasi negativi e dei lieviti come causa di "batteriemia ospedaliera CVC correlata" (soprattutto nell'A.chir e in UTI).

\section{M042 \\ ANALISI DELLA SENSIBILITA' AGLI ANTIBIOTICI NEI MICRORGANISMI ISOLATI DALLE EMOCOLTURE (200I-2002)}

Usiglio D., Casini-Lemmi M., Resta M.A., Locca M., Lanata M., Mori M.

Struttura Complessa di Analisi Chimico-Cliniche e Microbiologiche "E.O. Ospedali Galliera", Genova

Obiettivi Valutare la sensibilità agli antibiotici nei microrganismi causa di batteriemia significativa nel biennio (20012002). Rilevare le differenze tra isolati da batteriemie ospedaliere e comunitarie con particolare attenzione ai meccanismi di resistenza emergenti.

Metodi Batteriemie considerate significative erano quelle relative all'isolamento da emocoltura di un reale patogeno in relazione al numero dei prelievi eseguiti e alle evidenze cliniche. Sono state definite infezioni ospedaliere le batteriemie significative insorte almeno 72 ore dopo il ricovero ospedaliero, non manifeste clinicamente ne in incubazione al momento dell'ammissione. Per l'esecuzione dell'antibiogramma è stato utilizzato routinariamente il sistema Sceptor (Becton Dickinson) con pannelli breakpoint, per isolati particolari e verifiche dei meccanismi di resistenza sono stati utilizzati: antibiogrammi manuali (Kirby-Bauer), MIC con E-Test (Biodisk), oxacillin e vancoscreen agar.

Risultati e Conclusioni Complessivamente sono stati evidenziati buoni livelli di sensibilità, nonostante la necessaria attenzione verso alcuni meccanismi di resistenza(Esbl, Vancoresistenza, Meticillinoresistenza). Si è osservata spesso una minor sensibilità agli antibiotici nei ceppi comunitari rispetto agli ospedalieri, spiegabile in parte considerando le provenienze dalle spedalizzazioni territoriali o dalle istituzioni per lungodegenti, l'aumento delle cateterizzazioni e dei turnover dei ricoveri, tutti elementi che favoriscono la circolazione di ceppi "ospedalieri" anche in comunità.

Nelle Enterobacteriaceae, carbapenemici e amikacina sono risultati gli antibiotici con le percentuali più alte di sensibilità. In Proteus si evidenzia maggiormente il fenomeno dei ceppi produttori di Esbl (35\%degli isolati) associata a multiresistenza. Nei bacilli gram negativi non fermentanti si è osservato alte percentuali di sensibilità ai carbapenemici e amikacina, livelli inferiori per ceftazidime. Negli Stafilococchi aurei la meticillino resistenza $(60 \%$ nei ceppi ospedalieri) e in misura minore negli Stafilococchi coagulasi negativi (meticillino resistenza $77 \%$ nei ceppi ospedalieri) si è rilevata spesso multiresistenza associata per macrolidi, aminoglicosidi fluorochinolonici e clindamicina. Tutti gli stafilococchi mostravano elevate percentuali di sensibilità ai glicopeptidi .Tutti gli Streptococchi isolati hanno dimostrato buone percentuali di sensibilità agli antibiotici testati (soprattutto beta-lattamici). Per quanto riguarda gli Enterococchi sia E.faecalis che E.faecium mantengono alte percentuali di sensibilità per i glicopeptidi (nonostante l'aumento degli isolamenti di ceppi VRE ) e per gli aminoglicosidi. 